OAI-PMH: http://www.indteca.com/ojs/index.php/Revista Scientific/oai

Artículo Original / Original Article

\title{
Categorías emergentes que conforman a la educación familiar a partir de la hermeneusis
}

\author{
Autor: Claudio Alejandro Castillo Esqueda \\ Universidad Nacional Experimental de la Seguridad, UNES \\ castilloclaudio200@gmail.com \\ Aragua, Venezuela \\ https://orcid.org/0000-0002-0158-7128
}

Resumen

Los seres humanos a través de la historia se han conformado en grupos sociales que les ha permitido sobrevivir a las diversas circunstancias a los cuales se enfrentan. Hordas, clanes, tribus, fueron evolucionando hasta llegar a conformarse la agrupación social conocida como la familia. Es esta agrupación, la que ha logrado permanecer por más tiempo en la historia de la humanidad. Como objetivo se busca obtener conocimientos de la educación familiar a partir de las interpretaciones de tres informantes claves. Una madre, un padre y un hijo. Usando para ello el método Hermenéutico, correspondiente al paradigma interpretativo y bajo este enfoque cualitativo se aprecian las posturas que se posee de la educación familiar, permitiendo construir categorías que la definan. Es por lo que considerar a la educación familiar como informal, sería como reconocer de informal a la misma familia, a sus integrantes y a lo que representa la familia para la sociedad.

Palabras clave: crianza del niño; educación no formal; sistema; niveles de enseñanza.

Código de clasificación internacional: 7202.02 - Hermenéutica.

\footnotetext{
Cómo citar este artículo:

Castillo, C. (2021). Categorías emergentes que conforman a la educación familiar a partir de la hermeneusis. Revista Scientific, 6(20), 79-91, e-ISSN: 2542-2987. Recuperado de: https://doi.org/10.29394/Scientific.issn.2542-2987.2021.6.20.4.79-91
}

Fecha de Recepción: 08-12-2020
Fecha de Aceptación: 16-03-2021
Fecha de Publicación: 05-05-2021 


\title{
Artículo Original / Original Article
}

\section{Emerging categories that make up family education from hermeneusis}

\begin{abstract}
Human beings throughout history have been conformed into social groups that have allowed them to survive the various circumstances they face. Hordes, clans, tribes, were evolving until the social group known as the family was formed. It is this group that has managed to remain the longest in the history of mankind. The objective is to obtain knowledge of family education from the interpretations of three key informants. A mother, a father and a son. Using the Hermeneutical method, corresponding to the interpretive paradigm and under this qualitative approach, the positions of family education are appreciated, allowing the construction of categories that define it. This is why considering family education as informal would be like recognizing the family itself, its members and what the family represents for society as informal.
\end{abstract}

Keywords: child rearing; informal education; system; teaching levels. International classification code: 7202.02 - Hermeneutics.

\section{How to cite this article:}

Castillo, C. (2021). Emerging categories that make up family education from hermeneusis. Revista Scientific, 6(20), 79-91, e-ISSN: 2542-2987. Recovered from: https://doi.org/10.29394/Scientific.issn.2542-2987.2021.6.20.4.79-91

Date Received:

08-12-2020
Date Acceptance: 16-03-2021
Date Publication: 05-05-2021 


\section{Introducción}

La incidencia de la educación familiar en el proceso de conformación de la personalidad y de las virtudes sociales debe ser considerada con la relevancia que le corresponde. En virtud de la incidencia que este proceso tiene hacia la sociedad y viceversa. De acuerdo con Martínez, Anaya y Salgado (2014): es fundamental comprender desde diversos planteamientos teóricos lo esencial que es para el desarrollo del ser humano la educación familiar y que la misma no puede ser vista o considerada como de informal.

En este sentido se requiere concientizar de la importancia que tiene la educación familiar y que la misma representa las construcciones de moldes sociales y como lo expresa Hernández (2005): "el tipo de molde que construyamos va a determinar que miremos el mundo y la vida de una forma o de otra. Pero lo importante es que esa construcción depende, en gran medida, de cómo sea la educación familiar recibida" (pág. 21).

Evidentemente, la sociedad incide sobre la formación del ser, a partir de la educación que se le imparta al mismo desde la familia, pero la familia, también tiene la posibilidad de incidir sobre la sociedad en la cual está circunscrito este ser humano.

Sin duda alguna, se requiere una perspectiva distinta para apreciar lo determinante que es la educación familiar en la construcción de sociedades prosperas y armoniosas. Las investigaciones o estudios científicos del proceso de la formación del ser social, como lo es la educación familiar, generan elementos de asaz relevancia, al permitirnos obtener información que sea capaz de direccionar el rumbo de la sociedad, a partir de orientar el origen de quienes la integren en un futuro.

Este artículo referente a la educación familiar plantea la necesidad de que sea reconocido como un proceso determinante capaz de la conformación de sociedades de paz; y que para ello debe ser vista como un verdadero proceso de educación y no considerarla como informal. Ya que considerarla 
informal, estaríamos considerando de informal a la misma familia, y por supuesto a sus integrantes. Estando en presencia de un desconocimiento real de lo que es el ser humano. Ya que todo proceso ontológico del ser debe ser respetado, como al mismo ser que lo efectúa.

En este mismo orden de ideas, el presente artículo no solo manifiesta su intención de defender y promover la educación familiar como un proceso determinante para la construcción de sociedades de paz, sino que plantea de igual manera, que se reconozcan a este poderoso proceso de la educación familiar, como parte de los derechos humanos que debe ser respetado, y que la misma no se le reconozca como informal. Ya que, si se irrespeta lo que desarrolla las capacidades en una persona, entonces se irrespeta a la persona misma. De igual manera este proceso educativo es un motor capaz de construir a una nación de paz, a un país próspero a partir de la formación del recurso más valioso que existe: el ser humano.

Es así, que se platea entonces como objetivo la necesidad de conocer la interpretación que se tiene de un elemento tan fundamental como lo es la educación familiar y obtener conocimientos que nos permitan comprender la importancia de esta, para el desarrollo adecuado de las futuras generaciones.

\section{Metodología}

Esta investigación se enmarca en un enfoque cualitativo bajo el paradigma interpretativo. Para llegar hasta ese saber requerido, se establece que el método de la investigación es el hermenéutico, ya que los fenómenos sociales siempre van a requerir una constante hermeneusis. No es por la falta de confiabilidad de sus resultados, sino que el centro desde donde sale los saberes son circunstancias naturales y no controladas, y el contexto humano cambia tan necesariamente sea lo requerido para adaptarse o sobrevivir a nuevos retos sociales.

Es así como adentrarse en la realidad requiere de un modo ser y de 
andar, para poder llegar así hasta ella. El abordaje a lo investigado depende del método a utilizar, pero más allá de cumplimientos de protocolos científicos, en realidad el método de investigación empleado permite comprender la visión filosófica en que se encuentra el investigador. Ya que el método posee algunas características peculiares que requieren de un compromiso personal, para lograr alcanzar los objetivos o propósitos en estudio.

Así mismo, el escenario de la investigación fue el municipio José Ángel Lamas del estado Aragua, de la república bolivariana de Venezuela. En cuanto a los informantes claves, se utilizaron tres: una madre, un padre y un hijo. Es necesario establecer que la visión que posee el investigador con su objeto de estudio es de manera positiva. Busca reconocer la buena labor, la importancia, los aspectos relevantes y determinantes de la educación familiar, como para no considerarla, como un mero proceso informal.

El método hermenéutico permite conocer la interpretación de esa realidad expresada por quienes la han inculcado y quienes la han recibido. Con respecto a la técnica de recolección de información, se implementó la entrevista y como instrumentos de recolección de la información, la entrevista estructurada.

A partir de la entrevista estructurada, se alcanzaron cinco categorías emergentes que permite apreciar la enjundia que representa la educación familiar. Las mismas son las siguientes: Modelación familiar, filosofía moral, proyección social, con-ciencia familiar y pensamiento comunitario.

\section{Resultados}

\subsection{Modelación familiar}

Es de vital importancia que exista una correspondencia entre lo que se enseña y en lo que se es como padre, madre, o como persona que está a cargo de la conducción de un grupo familiar. Al respecto, Osorio (2008): señala que "dentro del elemento cognitivo juega un papel importante el modelado. 
Para que el niño aprenda que determinada conducta es buena, es importante que la vea ejecutada por personas que él valora" (pág. 60). La estructura que determina lo que va a hacer un ser humano, se construye es a partir de la educación familiar.

Es así, que ella es la mayor influencia que tiene una persona como agente socializador. $Y$ es un agente, porque no se requiere de una madre o un padre, sino que aquella persona que suministre, enseñe, guíe, oriente, eduque a infantes, materializa la educación familiar. No requiere de un espacio físico, ni de reconocimiento legal para que se ejerza, existe y ya. Es multidimensional, ya que sus efectos se encuentran presentes en todas las sociedades. Somos, lo que la educación familiar ha hecho de nosotros.

De acuerdo con esto, es imposible desligar lo que es, lo que piensa, lo que siente el educador familiar y de lo que trasmite al nuevo integrante de la familia. Y no porque se crezca, se sea adulto, en una familia, deja de existir la educación familiar. Con el tiempo esta reafirma el modelamiento familiar que se le enseño cuando estaba en la etapa de la educabilidad.

\subsection{Esencia social}

Cada familia posee una filosofía, un articulado moral que le determina su dinámica, un para qué de su existencia. Quien ejerce la educación familiar posee una responsabilidad en cuanto a ese que hacer de la familia con respecto a la comunidad, a la sociedad.

Entonces, ese educador familiar posee una característica particular, y que Bernal (2008): al respecto señala como "la autoridad en la familia se constituye en quien tiene la competencia para decir que hay que hacer y eso se ejercita creando confianza, exigiendo y acompañando en la frustración que experimenta un hijo cuando le cuesta hacer algo" (pág. 16). Es de vital importancia reconocer la fuerza que posee esas decisiones que tome quien lleva la conducción del grupo familiar. 
La esencia social nos dice que somos como familia, que aportamos a la comunidad, a la sociedad. La educación familiar es tan importante, que ella determina la esencia de cada familia, ella orienta y guía lo que aportamos a nuestro país. De cómo vamos a reaccionar ante las vicisitudes de la vida. De cómo vamos a educar a nuestros hijos. La educación familiar establece nuestro futuro, y eso jamás puede ser considerado como informal.

\subsection{Proyección social}

Es necesario construir la personalidad que requiere los integrantes que conforman la familia. Si bien es cierto, existen familias que desconocen lo influyente que será la educación familiar sobre los educandos en un futuro, no por ello debe considerársele como informal. Este proceso educativo proyecta socialmente en quien lo recibe la esencia, el modelamiento, el nivel de consciencia y el pensamiento comunitario que posee la familia.

La proyección social es la representación cultural del momento histórico que vive esa familia. Ya que esa representación en la sociedad que ejercen las personas, no es más que la sumatoria de estamentos inculcados en su proceso educativo inicial. Los hechos históricos nos trasmiten como fueron educados y para que, quienes lo vivieron.

Sería entonces interesante preguntarse ¿Para que participe en qué, estoy educando a mi familia? La proyección social sin duda alguna está direccionada por la educación familiar. La educación disciplinada de Amy Chua, según Ge (2011): permite apreciar la influencia de este poderoso proceso. Aunque puede generar mucha polémica el cómo se efectúa, sin embargo, es mejor tener el control de la conducción del destino de las generaciones venideras por parte de la familia, a permitir que sea direccionada por los medios de comunicación de masas. 


\subsection{Consciencia familiar}

La realidad en la que se desarrolla nuestra vida, posee una cantidad indefinida de variables, de factores, de hechos, de circunstancias, que no podemos controlar. Esa consciencia familiar de esta variada realidad se enseña progresivamente a los integrantes de la familia a medida que estos vayan creciendo, puedan comprender de que se trata y si en realidad es pertinente que se conozca de esta parte de la realidad en donde se habita. El educador familiar debe conocer las variedades de la realidad en la que los integrantes de su familia hacen vida.

Por ende, conoce de los peligros, de la violencia, de los riesgos que están presentes en su comunidad. Está consciente que debe enseñar a su familia acerca de los mismos. La educación familiar es un medio que salva vidas. Es a partir de ella que las personas conocen lo que representa un peligro para ellas. Es la verdadera prevención. Y se debe considerar como la base de la seguridad ciudadana. Ya que la educación familiar, es quien establecerá cuales son las necesidades y como satisfacerlas.

La perspectiva que debe poseer la educación familiar debe ser holística, al respecto Runte-Geidel (2015): expresa que "esta tarea educadora de la familia se da desde una perspectiva de la educación integral [...]" (pág. 21). Es imposible pensar que educar en valores solamente es educar en familia, para la familia, en sociedad y para la sociedad. La visión compleja de la realidad debe ser considerada en la modernidad actual.

\subsection{Pensamiento comunitario}

Pensarse individual rodeado por cientos de familias, es estar totalmente a alejado de lo que es vivir en familia. La unión o acercamiento por intereses comunes por parte de integrantes de familias en un espacio residencial determinado, esto se conoce como pensamiento comunitario. Sin importar la esencia social de las familias, si existen para bien o para mal, todas poseen la 
necesidad de un parentesco. No es que se parecen y se junta. Se unen por la necesidad de parecerse, ya que en realidad una familia no puede estar apartada de otras. Esa forma de habitad, es uno de sus elementos ontológicos.

La influencia de educar en familia y lograr obtener lo que para esa comunidad significa necesidad. Hace que las otras familias reproduzcan el modelo de educación familiar que esa familia exitosa logro alcanzar. Ahora bien, si esa familia exitosa no vive en la comunidad, de igual manera pertenecemos a una filiación ya sea por la televisión, redes sociales, actores, deportistas, emprendedores, comerciantes, empresarios, en fin, la familia busca su reflejo en la sociedad en la que quiere pertenecer. Y es la educación familiar la que nos genera ese pensamiento comunitario, y es ella, la educación familiar, la que nos mantiene unidos como sociedad. Porque al final, la sociedad no es más que millones de familias unidas, ejerciendo las voluntades aprendidas por la educación familiar que recibieron.

Es fundamental reconocer que, así como se identifican familias entre sí, y comparten actividades para hacer el bien, también tenemos a familias discentradas, que lastimosamente sus integrantes se reúnen para perjudicar a su contexto. Ese pensamiento comunitario de las familias, está determinado por la educación familiar que reside en ellos. Algunos han sido víctimas de situaciones incorrectas en su proceso educativo.

Al respecto, Martín (2007): refiere que "son un conjunto de alteraciones significativas y extremadamente dañinas de la dinámica familiar que impiden el cumplimiento adecuado de la función educativa [...]" (pág. 78). De ahí la atención que se le debe prestar a lo que realmente significa la educación familiar y que la misma debe ser considerada al planificarse acciones, programas, proyectos, planes y por supuesto, políticas públicas.

A continuación, se presentan en la tabla 1, las categorías emergentes obtenidas a partir de la hermeneusis y sus principales características: 
Tabla 1. Categorías emergentes obtenidas a partir de la hermeneusis.

\begin{tabular}{|l|l|}
\hline Categorías Emergentes & \multicolumn{1}{|c|}{ Principales Características } \\
\hline Modelación familiar & $\begin{array}{l}\text { Quien educa es quien modela. El principal } \\
\text { socializador es quien educa. Por ser adulto no se } \\
\text { pierde la modelación. }\end{array}$ \\
\hline Esencia social & $\begin{array}{l}\text { Es la filosofía de la familia, lo que aportamos a la } \\
\text { sociedad, es el articulado moral de la familia. }\end{array}$ \\
\hline Proyección social & $\begin{array}{l}\text { Es la representación cultural del momento histórico } \\
\text { que vive esa familia. Vamos familiarmente al lugar } \\
\text { de dónde venimos. }\end{array}$ \\
\hline Consciencia familiar & $\begin{array}{l}\text { Conocimiento de los elementos presentes en la } \\
\text { realidad en la que están circunscritos. }\end{array}$ \\
\hline Pensamiento comunitario & $\begin{array}{l}\text { Se educa para poder estar en sociedad, esto no se } \\
\text { puede alcanzar realmente, sino se está dentro del } \\
\text { pensamiento comunitario. }\end{array}$ \\
\hline
\end{tabular}

Fuente: El Autor (2021).

\section{Conclusiones}

Es necesario reconocer la importancia que representa la educación familiar, gracias a ella, los saberes, conocimientos, costumbres, tradiciones, hábitos, creencias, capacidades y demás. Es decir, gracias a la educación familiar, ha sido de generación en generación, parte del legado cultural de la humanidad.

El reconocimiento de informal que se le asigna a este proceso educativo e histórico. Y que ha sido más antiguo que la misma escuela. Es por mostrar un desconocimiento de los aspectos trasmisores de la cultura y de la historia.

Las diversas disciplinas científicas, tiene un aliado para la trasmisión de sus saberes, a través de este proceso educativo. $Y$ que es capaz de generar un conocimiento tan cierto, como lo es la misma realidad en la que participa.

La sociedad puede ser direccionada, a partir de un paradigma familiar, en donde la educación familiar sea el medio para alcanzar los propósitos. No existe argumentación alguna, que pueda demostrar la informalidad de un proceso, que ha hecho que millones de seres humanos participen en la experiencia más maravillosa que hay, vivir en familia. 
Artículo Original / Original Article

\section{Referencias}

Bernal, A. (2008). Autoridad y educación familiar: portaciones desde la psicología aplicada a la familia. International Journal of Developmental and Educational Psychology, 4(1), 13-22, e-ISSN: 02149877. Recuperado de:

https://www.redalyc.org/articulo.oa?id=349832319001

Ge, L. (2011). Todo sobre la "madre tigre": un prisma-polígono de la cultura tradicional de la educación familiar de China. México y la Cuenca del Pacífico, (42), 97-122, e-ISSN: 1665-0174. Recuperado de: https://www.redalyc.org/articulo.oa?id=433747500005

Hernández, P. (2005). ¿La Educación Familiar puede Configurar los moldes Mentales o la Inteligencia Emocional de los Hijos?. International Journal of Developmental and Educational Psychology, 1(1), 17-38, e-ISSN: 0214-9877. Recuperado de:

https://www.redalyc.org/articulo.oa?id=349832486001

Martín, M. (2007). Los Trastornos Psicosociales de la Educación Familiar. Alternativa pedagógica para su mejoramiento desde la escuela. EduSol, 7(18), 70-85, e-ISSN: 1729-8091. Recuperado de: https://www.redalyc.org/articulo.oa?id $=475748657008$

Martínez, C., Anaya, M., \& Salgado, D. (2014). Desarrollo de la personalidad y virtudes sociales: relaciones en el contexto educativo familiar. Educación y Educadores, 17(3), 447-467, e-ISSN: 0123-1294. Recuperado de:

https://www.redalyc.org/articulo.oa?id=83433781003

Osorio, A. (2008). Información, afecto y voluntad en la educación familiar del altruismo. International Journal of Developmental and Educational Psychology, 4(1), 59-64, e-ISSN: 0214-9877. Recuperado de: http://www.redalyc.org/articulo.oa?id=349832319005

Runte-Geidel, A. (2015). La pedagogía familiar y la educación familiar 
como contextos teóricos de la asignatura educación y familia. Revista Internacional de apoyo a la inclusión, logopedia, sociedad y multiculturalidad, 1(1), 11-23, e-ISSN: 2603-9443. Recuperado de: https://www.redalyc.org/articulo.oa?id=574661394002 


\section{Artículo Original / Original Article}

\section{Claudio Alejandro Castillo Esqueda \\ e-mail: castilloclaudio200@gmail.com}

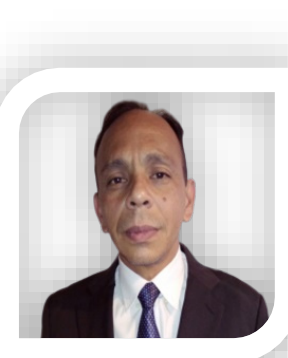

Nacido en Cagua, estado Aragua, Venezuela, el 5 de marzo del año 1972. MSc. en Seguridad Ciudadana de la Universidad Nacional Experimental de la Seguridad (UNES); Lcdo. en Educación, mención Desarrollo Cultural de la Universidad Nacional Experimental Simón Rodríguez (UNESR); docente en la Universidad Nacional Experimental de la Seguridad (UNES); Jefe de Área de Conocimiento de Habilidades de la Comunicación Oral y Escrita; docente de Aprender a Aprender; Humboldt County Office of Education; Historia de la seguridad; Policía comunal; Plan de Desarrollo Nacional; Proyecto socio integrador, entre otras; docente del diplomado en seguridad ciudadana y servicio penitenciario; Participante en 4 congresos regionales de seguridad ciudadana; 2 congresos pedagógicos y 1 congreso nacional en seguridad ciudadana.

El contenido de este manuscrito se difunde bajo una Licencia de Creative Commons ReconocimientoNoComercial-Compartirlgual 4.0 Internacional 